\title{
Patología dual en Depresión: \\ recomendaciones en el tratamiento
}

\section{Dual diagnosis in Depression: \\ treatment recommendations}

\author{
Judit Tirado-Muñoz*; Adriana Farré***; Joan Mestre-Pintó*; Nestor Szerman***; \\ MARTA TORRENS $* *, *, * * * *$.
}

* IIMIM-Institut Hospital del Mar d'Investigacions Mèdiques, Barcelona, Spain; ** Institut de Neuropsiquiatria i Addiccions, Parc de Salut Mar de Barcelona, Barcelona, Spain; *** Servicio de Psiquiatría. Hospital Gregorio Marañón. Madrid, Spain; **** Departament de Psiquiatria. Universitat Autònoma de Barcelona, Barcelona, Spain.

\section{Resumen}

La comorbilidad entre los trastornos por uso de sustancias (SUD) y la depresión mayor (DM) es la patología dual más común en el campo de las adicciones a sustancias, con prevalencias que oscilan entre el 12 y el $80 \%$ complicando la respuesta al tratamiento y empeorando el pronóstico de los pacientes. Diferenciar entre el diagnóstico de episodios depresivos inducidos y episodios depresivos primarios concurrentes al uso de sustancias es especialmente relevante para el manejo terapéutico.

En este artículo se presenta el estado actual de los tratamientos farmacológicos disponibles hasta el momento para la depresión comórbida en pacientes con SUD, teniendo en cuenta la seguridad y el potencial de abuso de los fármacos antidepresivos.

Debido a que la comorbilidad de DM y SUD es frecuente y a que estos pacientes presentan mayor gravedad psicopatológica y peor funcionamiento social, es crucial un modelo de tratamiento integrado y no abordar el tratamiento por separado.

Palabras clave: Depresión; Patología dual; Comorbilidad; Tratamiento; Recomendaciones.

\begin{abstract}
Comorbidity between substance use disorders (SUD) and major depression (MD) is the most common dual pathology in the field of addiction to substances and has prevalence rates ranging between $12 \%$ and $80 \%$, which complicates the response to treatment and worsens the prognosis of patients.

Differentiating between diagnoses of induced depressive episodes and primary depressive episodes concurrent to substance use is especially relevant for therapeutic management.

This article presents the state of the art of the currently available pharmacologic treatments of comorbid depression in patients with SUD, taking into account the safety and risk of abuse of antidepressant drugs.

Due to the fact that comorbidity of MD and SUD is frequent and presents greater psychopathological and medical severity, as well as worse social functioning, it is crucial to treat MD and SUD simultaneously using the integrated treatment model and not to treat both conditions separately.

Keywords: Depression; Dual pathology; Comorbidity; Treatment; Recommendations.
\end{abstract}


Los trastornos del estado de ánimo y los trastornos de ansiedad son los trastornos mentales asociados con mayor frecuencia con los trastornos relacionados con el consumo de sustancias (TUS) (San, Arranz, y Grupo de Expertos de la Guía de Práctica Clínica de Patología Dual, 2016). En esta revisión presentamos una puesta al día del conocimiento sobre la comorbilidad entre la depresión y los TUS, y las recomendaciones terapéuticas derivadas. A lo largo de la revisión para indicar la presencia simultánea de un episodio de DM y un TUS se utilizan de modo intercambiable los términos depresión dual, depresión comórbida con TUS o DM + TUS.

En los distintos estudios la prevalencia de esta asociación oscila entre el $12 \%$ y el $80 \%$. De acuerdo con Torrens y Rossi (2015), diversos factores explican este amplio rango. Entre los factores a considerar destacan: la sustancia principal de consumo (tabaco, alcohol, cocaína, opiáceos, hipnosedantes...); si el estudio se ha realizado en población general o en una muestra de consumidores -y en este último caso, si se han reclutado en centros de tratamiento de adicciones, en recursos asistenciales de salud mental o en otras poblaciones (prisión, entre "sin techo")-, y aspectos metodológicos tales como los criterios diagnósticos (DSM o CIE, en sus distintas versiones) o los instrumentos diagnósticos utilizados (entrevistas diagnósticas, como la PRISM, SCID o SCAN, o de cribado, como la ECDD).

En una revisión sistemática con metaanálisis de los estudios epidemiológicos en población general, realizados entre 1990 y 2014, los autores confirman la elevada asociación entre DM y TUS (Lai, Cleary, Sitharthan, y Hunt, 2015). Esta asociación es mayor para el consumo de drogas ilegales que para el alcohol y más elevada para los trastornos con criterios de dependencia que para los trastornos por abuso, independientemente del criterio temporal para establecer la prevalencia (a lo largo de toda la vida o en los últimos 12 meses). En la Tabla 1 se describen los principales resultados.

En poblaciones clínicas en los distintos recursos asistenciales y en algunas poblaciones especiales (por ej. personas en prisión o "sin techo"), la prevalencia a nivel europeo de la comorbilidad de DM y TUS está disponible en diversas publicaciones como el Insight 19 del Observatorio Europeo de las Drogas y las Toxicomanías (EMCDDA, 2015) y en otras (Arias et al., 2013; Torrens y Rossi, 2015).

Tabla 1. Prevalencia TUS-DM en estudios epidemiológicos en población general (Lai y cols, 2015).

\begin{tabular}{lll}
\hline \multirow{2}{*}{$\begin{array}{l}\text { DM } \\
\text { asociada }\end{array}$} & Alcohol & $\begin{array}{l}\text { Abuso OR 1.53, 95\% Cl 1.20-1.95 } \\
\text { Dependencia OR 3.09, 95\% Cl 2.38-4.03 }\end{array}$ \\
\cline { 2 - 3 } & Otras drogas & $\begin{array}{l}\text { Abuso OR 3.80, 95\% Cl 3.02-4.78 } \\
\text { Dependencia OR 4.83, 95\% Cl 3.01-7.73 }\end{array}$ \\
\hline
\end{tabular}

Nota. TUS: trastornos por uso de sustancias; DM: Depresión Mayor; OR: Odds Ratio $\mathrm{Cl}$ : Intervalo de confianza.
Además tanto los estudios realizados en población general como los realizados en población clínica indican que la DM comórbida con TUS es más frecuente en mujeres que en hombres, y es dos veces más frecuente que en las mujeres de población general, por lo que las mujeres con TUS constituyen un colectivo especialmente vulnerable (Torrens et al., 2011).

\section{Etiopatogenia}

Se proponen tres hipótesis para explicar la elevada concurrencia de DM y TUS:

1.TUS y DM comparten factores de riesgo comunes, como acontecimientos vitales estresantes, traumas psicológicos, vulnerabilidad genética y/o alteraciones neurobiológicas previas que conducen a la aparición de ambos trastornos, sin que exista una relación causal entre ellos.

2. El consumo continuado de algunas sustancias de abuso conlleva cambios neurobiológicos a través de mecanismos neuroadaptativos que median la DM.

3. El TUS se desarrolla para aliviar la DM (hipótesis de la automedicación). En este caso, la DM incrementa las conductas deriesgo de consumo.

Tanto en la DM como en los TUS, los factores genéticos y ambientales son cruciales en la facilitación de mecanismos neurobiológicos relacionados con su psicopatogenia (Brady y Sinha, 2005; Schuckit, 2006). Los principales mecanismos neuronales y moleculares implicados en la neurobiología de la depresión incluyen el sistema monoaminérgico, el eje hipotálamo-hipofisario-adrenal, el sistema immunológico, factores neurotróficos, el sistema endocannabinoide, el ritmo circadiano y el sistema de control de ingesta y metabolismo (Belmaker y Agam, 2008; Krishnan y Nestler, 2010; Valverde y Torrens, 2012; Valverde et al., 2009).

La mayoría de estos sistemas también están implicados en el desarrollo de los TUS (Brady y Sinha, 2005; Gutiérrez-Sacristán et al., 2015; Valverde y Torrens, 2012). Por otra parte, los circuitos de recompensa, de los más relevantes en la patogenia de la adicción (Wise, 1989), también están implicados en la neurobiología de los trastornos depresivos (Nestler y Carlezon, 2006).

\section{Aspectos clínicos}

La identificación clínica de la DM en consumidores de sustancias es compleja debido a distintos factores. Por una parte, los efectos agudos o crónicos del consumo de sustancias pueden mimetizar los síntomas depresivos, dificultando la diferenciación entre los síntomas propios de una DM independiente de los síntomas relacionados con el consumo o de su abstinencia. Por otra parte, los diagnósticos psiquiátricos, como el de la DM, son más sindrómicos que enfermedades con una fisiopatología clara y con marcadores biológicos asociados. Esta ausencia de marcadores biológicos ha obligado a los psiquiatras a desarrollar criterios 
diagnósticos operativos, incluyendo los del DSM y los del CIE, y a diseñar entrevistas diagnósticas clínicas para mejorar la validez y fiabilidad de los diagnósticos psiquiátricos. En referencia al diagnóstico de otros trastornos psiquiátricos en consumidores de sustancias, los criterios utilizados han ido cambiando hasta llegar a los criterios del DSM-IV (American Psychiatric Association, 1994) y mantenidos en el DSM-IV-TR (American Psychiatric Association, 2002) y el DSM-5 (American Psychiatric Association, 2013), reconociendo la necesidad de considerar tres condiciones para facilitar un diagnóstico más preciso:

- "Efectos esperables": son los síntomas considerados propios de la intoxicación o la abstinencia de una sustancia determinada, y por tanto no deben ser tenidos en cuenta como síntomas para diagnosticar depresión (ej. insomnio durante la intoxicación aguda por estimulantes o, durante el periodo de abstinencia de opiáceos).

- "Inducidos por sustancias": trastornos que aparecen en relación con el consumo o la abstinencia de la sustancia, pero considerados como excesivos en relación a los efectos esperados.

- "Primarios": trastornos mentales que no son inducidos por sustancias ni debidos a una enfermedad médica, es decir, son trastornos independientes.

Los profesionales suelen recordar más el concepto de trastorno primario o independiente y el de trastorno inducido, que el concepto "efecto esperable", que sin embargo es muy relevante para poder aumentar la validez y fiabilidad diagnóstica.

En la práctica clínica la diferenciación entre los episodios depresivos primarios y los inducidos por el consumo constituye una de las dificultades para la realización del diagnóstico del cuadro depresivo cuando hay coocurrencia de consumo de sustancias. Para ello hay disponibles distintas entrevistas diagnósticas que ayudan a establecer el diagnóstico. Entre ellas, la Psychiatric Research Interviewfor Substance and Mental Disorders (PRISM) (Hasin et al., 1996) permite establecer diagnóstico de depresión primaria o inducida por sustancias de manera válida y fiable. Esta diferencia puede ser especialmente relevante para el manejo terapéutico. En la Tabla 2 se describen los principales indicadores clínicos que ayudan al diagnóstico diferencial entre los episodios depresivos inducidos y los episodios depresivos primarios concurrentes al consumo de sustancias.

Se ha observado que en el caso de los TUS por cocaína, opiáceos o en policonsumidores el episodio de DM suele ocurrir con mayor frecuencia de forma independiente del consumo (Torrens, Gilchrist, y Domingo-Salvany, 2011) mientras que en el caso del alcohol se ha descrito una mayor prevalencia de asociación con DM inducida (Schuckit, Smith, y Kalmijn, 2013). Sin embargo se pueden encontrar ambos tipos de depresiones (primarias e inducidas) en el mismo paciente (Langås, Malt, y Opjordsmoen, 2013; To-
Tabla 2. Indicadores clínicos para el diagnóstico de un episodio depresivo concurrente al consumo de sustancias.

\begin{tabular}{ll}
\hline Depresión Primaria & Depresión Inducida \\
\hline $\begin{array}{l}\text { La sintomatología depresiva } \\
\text { aparece durante un fase de } \\
\text { consumo estable }\end{array}$ & $\begin{array}{l}\text { Aparición de la sintomatología } \\
\text { depresiva durante una escalada } \\
\text { de consumo }\end{array}$ \\
\hline $\begin{array}{l}\text { La sintomatología depresiva } \\
\text { persiste después de un periodo } \\
\text { de abstinencia }\end{array}$ & $\begin{array}{l}\text { Aparición de la sintomatología } \\
\text { depresiva durante una disminución } \\
\text { importante del consumo }\end{array}$ \\
\hline $\begin{array}{l}\text { Antecedentes de episodio } \\
\text { depresivo en ausencia de consumo } \\
\text { de sustancias }\end{array}$ & \\
\hline $\begin{array}{l}\text { Antecedentes de buena respuesta } \\
\text { a tratamientos antidepresivos }\end{array}$ & \\
\hline $\begin{array}{l}\text { Antecedentes familiares } \\
\text { de depresión }\end{array}$ & \\
\hline
\end{tabular}

Tabla 3. Principales características clínicas de la depresión dual.

\begin{tabular}{l}
\hline Características clínicas de la depresión dual \\
\hline La DM dual es más frecuente cuando hay un TUS de gravedad moderada- \\
grave, que si el TUS es de gravedad leve (en criterios DSM-IV la DM \\
dual es más frecuente en los trastornos por dependencia que en los \\
trastornos por abuso) \\
\hline La DM dual es más frecuente independiente que inducida (excepto en el \\
caso del TUS por alcohol) \\
\hline La presencia de DM (primaria o Inducida) se asocia con un curso \\
desfavorable del TUS \\
\hline La presencia de TUS se asocia a un curso desfavorable de la DM \\
\hline Los pacientes con depresión dual presentan mayor prevalencia de \\
intentos de suicidio/suicidios consumados \\
\hline Los pacientes con depresión dual presentan más comorbilidades \\
médicas y psiquiátricas (incluyendo más TUS) \\
\hline Los pacientes con depresión dual presentan mayor problemática \\
social y más utilización de recursos sanitarios, incluyendo más \\
hospitalizaciones psiquiátricas
\end{tabular}

rrens, Gilchrist, y Domingo-Salvany, 2011). Por otra parte, se ha observado que los pacientes con DM tienen el doble de posibilidades de desarrollar un TUS y que los pacientes que presentan un TUS tienen el doble de riesgo de padecer una DM a lo largo de su vida (Boden y Fergusson, 2011). Además, la coexistencia de ambos trastornos se ha asociado a un curso desfavorable de ambas patologías, con peor respuesta al tratamiento y peor pronóstico (Agosti y Levin, 2006; Davis, Uezato, Newell, y Frazier, 2008). Así, en estudios de seguimiento en muestras de pacientes dependientes de sustancias se ha observado que la presencia de episodios depresivos mayores, tanto primarios como inducidos, facilitaba la recaída en el consumo (Landheim, Bakken, y Vaglum, 2006; Samet et al., 2013). Asimismo, diversos estudios han observado que la comorbilidad del TUS en pacientes con DM aumenta la gravedad clínica de estos pacientes, existiendo un mayor riesgo de conductas suicidas (Marmorstein, 2011; Szerman Bolotner et al., 2011). Además, estos pacientes tienen mayor probabilidad de desarrollar otras comorbilidades médicas, lo que hace aún más difícil 
el tratamiento. Así pues, y como es esperable debido a su elevada gravedad clínica, estos pacientes duales presentan además una considerable gravedad psicosocial y realizan una mayor utilización de los recursos sanitarios, incluyendo los servicios de urgencias y las hospitalizaciones psiquiátricas (Martin-Santos et al., 2006; Mueller et al., 1994; Pettinati, O'Brien, y Dundon, 2013; Samet et al., 2013).

Así pues, con el conocimiento disponible, se puede afirmar que los episodios depresivos inducidos pueden ser tan o más graves que los primarios o independientes, tanto en cuanto a recaída en el consumo como en la gravedad de la sintomatología depresiva, incluyendo el riesgo suicida.

En la Tabla 3 se resumen las principales característicasclínicas de la depresión dual.

\section{Tratamiento de la depresión dual}

Dada la elevada frecuencia y la gravedad clínica y social de estos pacientes duales, es importante su tratamiento. Sin embargo, todavía disponemos de pocos estudios sobre el tratamiento de la depresión dual, y la mayoría de ellos han sido realizados en pacientes con dependencia del alcohol. A continuación se presenta el estado actual del manejo clínico de los pacientes con DM y TUS.

\section{Recomendaciones generales}

1. Un episodio depresivo debe tratarse a pesar de que el paciente esté en situación de consumo activo. El tratamiento de la depresión dual debe tener en cuenta ambos trastornos; el tratamiento de la depresión no puede sustituir al tratamiento de la adicción.

2. Debe tratarse la adicción aunque el paciente se halle en un episodio depresivo. El tratamiento antidepresivo tiene un impacto limitado en el uso de sustancias; se debe plantear tratamiento concomitante específico para el TUS.

3. El uso de sustancias no es una limitación para el tratamiento de la depresión.

4. Los efectos de los antidepresivos son superiores cuando los pacientes presentan una DM primaria.

5. El tratamiento debe contemplar el abordaje farmacológico y el psicoterapéutico.

\section{Tratamiento farmacológico}

El enfoque terapéutico farmacológico de la DM con TUS debe contemplar no sólo la eficacia de los distintos fármacos, sino también aspectos de seguridad del uso de los antidepresivos, sus posibles interacciones con el consumo de las distintas sustancias y el potencial de abuso de los distintos fármacos administrados para el tratamiento de la depresión dual.

A continuación se describen brevemente los aspectos más relevantes que se deben tener en cuenta en la prescripción de los antidepresivos.

\section{Eficacia de los fármacos antidepresivos en la depresión dual}

En la actualidad se dispone de dos revisiones sistemáticas de ensayos clínicos controlados analizadas con metaanálisis (Nunes y Levin, 2004; Torrens, Fonseca, Mateu, y Farré, 2005). Los principales resultados fueron que los inhibidores selectivos de la recaptación de serotonina (ISRS) obtenían peores resultados que los antidepresivos no ISRS en el tratamiento de la DM dual y que los antidepresivos no afectan directamente a la mejoría del consumo de sustancias. Posteriormente se han publicado otros estudios sobre el tratamiento de la depresión dual. A continuación se resumen los siete estudios posteriores sobre la eficacia de los antidepresivos en el tratamiento de la DM comórbida con el trastorno por consumo de alcohol (Tabla 4) y los seis estudios posteriores sobre la eficacia de los antidepresivos en el tratamiento de la DM comórbida con el trastorno por consumo de cocaína (Tabla 5).

En cuanto a la eficacia del tratamiento antidepresivo en la depresión comórbida con el trastorno por consumo de opiáceos, hay que destacar que posterior a la revisión sistemática con metaanálisis de Torrens (Torrens et al., 2005), sólo se ha publicado una revisión de la Cochrane (Pani, Vacca, Trogu, Amato, y Davoli, 2010), en la que se incluyeron los mismos estudios. Posteriormente y hasta la actualidad no se ha publicado ningún otro estudio.

En el tratamiento de DM y trastorno por dependencia de cannabis se dispone de un único ensayo clínico aleatorizado controlado con placebo, en 103 pacientes con trastorno por uso de cannabis y DM o distimia, donde se comparó el efecto de venlafaxina de liberación retardada con placebo durante 12 semanas. Además, todos recibieron tratamiento concomitante con sesiones semanales de terapia cognitivo-conductual individual. No se encontraron diferencias significativas en cuanto a la clínica depresiva y se objetivó un incremento en el uso de cannabis en los pacientes del grupo de venlafaxina de liberación retardada (Levin et al., 2013).

Así pues, la revisión de la literatura disponible del tratamiento farmacológico de la depresión dual permite afirmar que:

Los ISRS son los antidepresivos más utilizados en los estudios y en ningún caso han mostrado eficacia para el tratamiento de la depresión comórbida con trastorno por uso de alcohol, cocaína u opiáceos.

Se dispone de escasos estudios con otros fármacos antidepresivos no ISRS, y en este caso las evidencias indican que: a) imipramina y desipramina son eficaces en la mejoría de la depresión en pacientes con DM y trastorno por uso de alcohol y desipramina en DM y trastorno por uso de cocaína; b) otros antidepresivos estudiados como venlafaxina, mirtazapina y nefazodona no han mostrado eficacia para mejorar la depresión dual.

Ningún antidepresivo ha demostrado eficacia en la reducción del consumo de sustancias. 
Tabla 4. Ensayos clínicos doble ciego y controlados sobre DM y Trastorno por Consumo de Alcohol incluidos y no incluidos en los metaanálisis previos.

\begin{tabular}{|c|c|c|c|c|c|c|c|}
\hline Autores & $\begin{array}{l}\text { Tipo } \\
\text { estudio }\end{array}$ & $\begin{array}{l}\text { Fármaco/s a } \\
\text { estudio }\end{array}$ & $\mathbf{N}$ & $\begin{array}{l}\text { Tiempo } \\
\text { estudio }\end{array}$ & $\begin{array}{l}\text { Tratamiento } \\
\text { concomitante }\end{array}$ & $\begin{array}{l}\text { Eficacia sobre } \\
\text { depresión }\end{array}$ & $\begin{array}{l}\text { Eficacia sobre consumo } \\
\text { sustancias }\end{array}$ \\
\hline $\begin{array}{l}\text { Altamura } \\
1990^{\star}\end{array}$ & EACP & Viloxazina & 27 & $12 \mathrm{sem}$ & $\begin{array}{l}4 \text { semanas de } \\
\text { ingreso seguidas } \\
\text { de tratamiento } \\
\text { ambulatorio }\end{array}$ & $\begin{array}{l}\text { Sí. Disminución de sintomatología } \\
\text { depresiva con diferencias } \\
\text { significativas entre ambos grupos }\end{array}$ & $\begin{array}{l}\text { Ambos grupos mejoran el } \\
\text { consumo de alcohol sin } \\
\text { diferencias significativas entre } \\
\text { los grupos }\end{array}$ \\
\hline $\begin{array}{l}\text { Mc Grath } \\
1996^{\star}\end{array}$ & EACP & Imipramina & 56 & $12 \mathrm{sem}$ & $\begin{array}{l}\text { TCC individual y } \\
\text { prevención de } \\
\text { recaídas }\end{array}$ & $\begin{array}{l}\text { Sí. Disminución de sintomatología } \\
\text { depresiva con diferencias } \\
\text { significativas entre ambos grupos }\end{array}$ & Ningún efecto \\
\hline $\begin{array}{l}\text { Mason } \\
1996^{\star}\end{array}$ & EACP & Desipramina & 22 & 24 sem & $\begin{array}{l}\text { Alcohólicos } \\
\text { anónimos }\end{array}$ & $\begin{array}{l}\text { Sí. Disminución de sintomatología } \\
\text { depresiva con diferencias } \\
\text { significativas entre ambos grupos }\end{array}$ & $\begin{array}{l}\text { Disminución del consumo con } \\
\text { diferencias significativas entre } \\
\text { ambos grupos }\end{array}$ \\
\hline $\begin{array}{l}\text { Cornelius } \\
1997^{\star}\end{array}$ & EACP & Fluoxetina & 51 & $12 \mathrm{sem}$ & $\begin{array}{l}\text { Psicoterapia de } \\
\text { soporte }\end{array}$ & $\begin{array}{l}\text { Sí. Disminución de sintomatología } \\
\text { depresiva con diferencias } \\
\text { significativas entre ambos grupos }\end{array}$ & $\begin{array}{l}\text { Disminución del consumo con } \\
\text { diferencias significativas entre } \\
\text { ambos grupos }\end{array}$ \\
\hline $\begin{array}{l}\text { Roy-Byrne } \\
200{ }^{\star}\end{array}$ & EACP & Nefazodona & 31 & $12 \mathrm{sem}$ & TCC grupo & $\begin{array}{l}\text { Sí. Disminución de sintomatología } \\
\text { depresiva con diferencias } \\
\text { significativas entre ambos grupos }\end{array}$ & $\begin{array}{l}\text { Disminución del consumo sin } \\
\text { diferencias entre ambos grupos }\end{array}$ \\
\hline $\begin{array}{l}\text { Pettinati } \\
2001^{\star}\end{array}$ & EACP & Sertralina & 29 & 14 sem & $\begin{array}{l}\text { Terapia de los } \\
12 \text {-pasos }\end{array}$ & $\begin{array}{l}\text { No. Sin diferencias entre ambos } \\
\text { grupos }\end{array}$ & $\begin{array}{l}\text { Sin diferencias entre ambos } \\
\text { grupos }\end{array}$ \\
\hline $\begin{array}{l}\text { Gual } \\
2003^{*}\end{array}$ & EACP & Sertralina & 46 & $24 \mathrm{sem}$ & $\begin{array}{l}2 \text { semanas de } \\
\text { abstinencia } \\
\text { después de la } \\
\text { desintoxicación }\end{array}$ & $\begin{array}{l}\text { No. Disminución de } \\
\text { sintomatología depresiva sin } \\
\text { diferencias significativas entre } \\
\text { ambos grupos }\end{array}$ & $\begin{array}{l}\text { Disminución del consumo sin } \\
\text { diferencias entre ambos grupos }\end{array}$ \\
\hline $\begin{array}{l}\text { Moak } \\
2003^{\star}\end{array}$ & EACP & Sertralina & 82 & $12 \mathrm{sem}$ & $\begin{array}{l}\text { TCC individual } \\
\text { para alcohol y la } \\
\text { depresión }\end{array}$ & $\begin{array}{l}\text { No. Disminución de } \\
\text { sintomatología depresiva sin } \\
\text { diferencias entre ambos grupos }\end{array}$ & $\begin{array}{l}\text { Disminución del consumo sin } \\
\text { diferencias entre ambos grupos }\end{array}$ \\
\hline $\begin{array}{l}\text { Hernández- } \\
\text { Àvila } 2004^{\star}\end{array}$ & EACP & Nefazodona & 41 & 10 sem & $\begin{array}{l}\text { Psicoterapia de } \\
\text { soporte }\end{array}$ & $\begin{array}{l}\text { No. Disminución de } \\
\text { sintomatología depresiva sin } \\
\text { diferencias entre ambos grupos }\end{array}$ & $\begin{array}{l}\text { Disminución del consumo sin } \\
\text { diferencias entre ambos grupos }\end{array}$ \\
\hline $\begin{array}{l}\text { Kranzler } \\
2006\end{array}$ & EACP & Sertralina & 328 & 10 sem & No & $\begin{array}{l}\text { No. Disminución de } \\
\text { sintomatología depresiva sin } \\
\text { diferencias entre ambos grupos }\end{array}$ & No \\
\hline $\begin{array}{l}\text { Altintoprak } \\
2008\end{array}$ & EAC & $\begin{array}{l}\text { Amitriptilina vs } \\
\text { Mirtazapina }\end{array}$ & 44 & $8 \mathrm{sem}$ & No & $\begin{array}{l}\text { No. Disminución de sintomas } \\
\text { depresivos sin diferencias entre } \\
\text { ambos fármacos. Mejor tolerancia } \\
\text { de la mirtazapina }\end{array}$ & $\begin{array}{l}\text { No } \\
\text { Ambos mejoran el craving por } \\
\text { alcohol }\end{array}$ \\
\hline $\begin{array}{l}\text { Muhonene } \\
2008\end{array}$ & EAC & $\begin{array}{l}\text { Memantina } \\
\text { vs Escitalopram }\end{array}$ & 80 & 2 años & No & $\begin{array}{l}\text { No. Ambos fármacos } \\
\text { disminuyeron síntomas } \\
\text { depresivos sin diferencias }\end{array}$ & No evaluado \\
\hline $\begin{array}{l}\text { Cornelius } \\
2009\end{array}$ & EACP & Fluoxetina & 40 & $12 \mathrm{sem}$ & $\begin{array}{l}\text { TCC estándar } \\
\text { y terapia } \\
\text { motivacional }\end{array}$ & $\begin{array}{l}\text { No. Ambos fármacos } \\
\text { disminuyeron síntomas } \\
\text { depresivos sin diferencias }\end{array}$ & $\begin{array}{l}\text { No } \\
\text { Ambos mejoran consumo }\end{array}$ \\
\hline $\begin{array}{l}\text { Petinatti } \\
2010\end{array}$ & EACP & $\begin{array}{l}\text { Setralina } \\
\text { vs Naltrexona } \\
\text { vs Sertralina + } \\
\text { Naltrexona } \\
\text { vs placebo }\end{array}$ & 170 & 14 sem & $\begin{array}{l}\text { Grupo placebo } \\
\text { TCC estándar } \\
\text { prevención } \\
\text { recaídas }\end{array}$ & $\begin{array}{l}\text { No. Sertralina + naltrexona } \\
\text { mejoría de la depresión al final } \\
\text { del estudio en comparación con } \\
\text { resto de grupos, sin significación }\end{array}$ & $\begin{array}{l}\text { Sertralina + naltrexona mejoran } \\
\text { abstinencia y alarga tiempo } \\
\text { hasta recaída }\end{array}$ \\
\hline $\begin{array}{l}\text { Adamson } \\
2015\end{array}$ & EACP & $\begin{array}{l}\text { Natrexona + } \\
\text { Citalopram } \\
\text { Vs Natrexona + } \\
\text { Placebo }\end{array}$ & 138 & 12 sem & No & $\begin{array}{l}\text { No. Disminución de } \\
\text { sintomatología depresiva sin } \\
\text { diferencias entre ambos grupos }\end{array}$ & $\begin{array}{l}\text { Disminución del consumo sin } \\
\text { diferencias entre ambos grupos }\end{array}$ \\
\hline $\begin{array}{l}\text { Foulds } \\
2015\end{array}$ & EACP & $\begin{array}{l}\text { Natrexona + } \\
\text { Citalopram } \\
\text { Vs Natrexona + } \\
\text { Placebo }\end{array}$ & 138 & $12 \mathrm{sem}$ & No & $\begin{array}{l}\text { No. Mejoría de las escalas en } \\
\text { depresión inducida aunque en } \\
\text { relación con disminución del } \\
\text { consumo sin poder determinar } \\
\text { un efecto significativo en el } \\
\text { tratamiento }\end{array}$ & $\begin{array}{l}\text { Mayor disminución del consumo } \\
\text { en depresión inducida que en } \\
\text { independiente }\end{array}$ \\
\hline
\end{tabular}

Nota. EACP: ensayo clínico aleatorizado controlado con placebo. EAC: ensayo clínico controlado sin placebo. No: no eficacia hallada. ISRS: inhibidores selectivos de la recaptación de serotonina AD: antidepresivos. TCC: terapia cognitiva conductual. *Estudios incluidos en metaanálisis anteriores. 
Tabla 5. Ensayos clínicos doble ciego y controlados sobre DM y Trastorno por Consumo de Cocaína incluidos y no incluidos en los metaanálisis previos.

\begin{tabular}{|c|c|c|c|c|c|c|c|}
\hline Autores & $\begin{array}{l}\text { Tipo } \\
\text { estudio }\end{array}$ & $\begin{array}{l}\text { Fármaco/s a } \\
\text { estudio }\end{array}$ & $\begin{array}{l}\mathbf{N} \\
\text { total }\end{array}$ & $\begin{array}{l}\text { Tiempo de } \\
\text { estudio }\end{array}$ & $\begin{array}{l}\text { Tratamiento } \\
\text { concomitante }\end{array}$ & $\begin{array}{l}\text { Eficacia sobre la } \\
\text { depresión }\end{array}$ & $\begin{array}{l}\text { Eficacia sobre consumo de } \\
\text { sustancias }\end{array}$ \\
\hline $\begin{array}{l}\text { Ziedonis } \\
1991^{\star}\end{array}$ & EACP & $\begin{array}{l}\text { Desipramina o } \\
\text { Amantadina }\end{array}$ & 14 & 12 & PMM & $\begin{array}{l}\text { Disminución de } \\
\text { sintomatología } \\
\text { depresiva }\end{array}$ & $\begin{array}{l}\text { Sí. Disminución del consumo } \\
\text { con diferencias entre ambos } \\
\text { grupos }\end{array}$ \\
\hline $\begin{array}{l}\text { Nunes } \\
1995^{*}\end{array}$ & EACP & Imipramina & 69 & 12 & $\begin{array}{l}\text { Counselling } \\
\text { Individual }\end{array}$ & No. Ningún efecto & $\begin{array}{l}\text { No. Disminución del consumo } \\
\text { sin diferencias entre ambos } \\
\text { grupos }\end{array}$ \\
\hline $\begin{array}{l}\text { Cornelius } \\
1998^{\star}\end{array}$ & EACP & Fluoxetina & 17 & 12 & Terapia de soporte & $\begin{array}{l}\text { No. Disminución } \\
\text { de sintomatología } \\
\text { depresiva sin } \\
\text { diferencias entre } \\
\text { ambos grupos }\end{array}$ & No \\
\hline $\begin{array}{l}\text { Schmitz } \\
2001^{*}\end{array}$ & EACP & Fluoxetina & 68 & 12 & $\begin{array}{l}\text { TCC y prevención de } \\
\text { recaídas }\end{array}$ & $\begin{array}{l}\text { No. Disminución } \\
\text { de sintomatología } \\
\text { depresiva sin } \\
\text { diferencias entre } \\
\text { ambos grupos }\end{array}$ & No \\
\hline $\begin{array}{l}\text { Gonzalez } \\
\text { 2003* }\end{array}$ & EACP & Desipramine & 56 & 12 & $\begin{array}{l}\text { Manejo de } \\
\text { contingencias }\end{array}$ & $\begin{array}{l}\text { No. No diferencias } \\
\text { significativas }\end{array}$ & No \\
\hline $\begin{array}{l}\text { MacDowell } \\
2005\end{array}$ & EACP & Desipramina & 111 & $12 \mathrm{sem}$ & $\begin{array}{l}\text { TCC estándar } \\
\text { prevención de } \\
\text { recaídas }\end{array}$ & $\begin{array}{l}\text { Sí. Mejoría clínica } \\
\text { depresiva en } \\
\text { pacientes en grupo } \\
\text { desimipramina }\end{array}$ & No \\
\hline $\begin{array}{l}\text { Ciraulo } \\
2005\end{array}$ & EACP & Nefazodona & 69 & $8 \mathrm{sem}$ & $\begin{array}{l}\text { Sesiones } \\
\text { counselling de } 1 \\
\text { hora }\end{array}$ & $\begin{array}{l}\text { No. Ambos grupos } \\
\text { mejoran sin } \\
\text { diferencias }\end{array}$ & No \\
\hline $\begin{array}{l}\text { Afshar } \\
2012\end{array}$ & EACP & Mirtazapina & 24 & $12 \mathrm{sem}$ & $\begin{array}{l}\text { Terapia de } \\
\text { prevención } \\
\text { de recaídas } \\
\text { manualizada }\end{array}$ & $\begin{array}{l}\text { No. Disminución } \\
\text { clínica depresiva en } \\
\text { ambos grupos }\end{array}$ & No \\
\hline $\begin{array}{l}\text { Oliveto } \\
2012\end{array}$ & EACP & Sertralina & 86 & $12 \mathrm{sem}$ & $\begin{array}{l}\text { TCC estándar } \\
\text { prevención de } \\
\text { recaídas }\end{array}$ & $\begin{array}{l}\text { No. No diferencias } \\
\text { significativas }\end{array}$ & No \\
\hline $\begin{array}{l}\text { Mancino } \\
2014\end{array}$ & EACP & $\begin{array}{l}\text { Sertralina } \\
\text { vs } \\
\text { Sertralina }+ \\
\text { Gabapentina }\end{array}$ & 99 & $12 \mathrm{sem}$ & $\begin{array}{l}\text { TCC estándar } \\
\text { prevención de } \\
\text { recaídas }\end{array}$ & $\begin{array}{l}\text { No. Mejoría en todos } \\
\text { los grupos }\end{array}$ & $\begin{array}{l}\text { Grupo con sertralina mayor } \\
\text { tiempo hasta recaída }\end{array}$ \\
\hline $\begin{array}{l}\text { Raby } \\
2014\end{array}$ & EACP & Venlafaxina & 130 & 8 sem & $\begin{array}{l}\text { Prevención } \\
\text { de recaídas } \\
\text { manualizada }\end{array}$ & No & No \\
\hline
\end{tabular}

Nota. EACP: ensayo clínico aleatorizado controlado con placebo. TCC: terapia cognitiva conductual. *Estudios incluidos en metánalisis anteriores

\section{Seguridad de los fármacos antidepresivos en la depresión dual}

Un aspecto especialmente relevante en el tratamiento farmacológico de la depresión dual es la posibilidad de interacciones farmacológicas entre los antidepresivos y las propias sustancias de abuso, los fármacos utilizados para el tratamiento del TUS o los fármacos utilizados para el tratamiento de otras comorbilidades médicas que pueda padecer el paciente (por ej. infección por virus de inmunodeficiencia humana o por virus de la hepatitis C). Destaca que, según el sistema de información de efectos adversos de la Food and Drug Administration (FDA), desde el 2000 la metadona es la segunda causa más frecuente de arritmia por fármacos después de dofetilide (Kao et al., 2013). Debido a que la metadona es uno de los fármacos más utiliza- dos en el tratamiento del trastorno por uso de opiáceos, se recomienda la revisión de las interacciones de la metadona de Chou (2014). En la Tabla 6 se resumen las interacciones más relevantes que deben tenerse en cuenta en el manejo clínico de la depresión dual. Hay que tener precaución especial con los inhibidores de la monoaminooxidasa (IMAO) por su interacción, con resultados fatales, con la tiramina de algunos alimentos o bebidas alcohólicas, estando también totalmente contraindicados con el consumo de estimulantes (cocaína, anfetaminas, metanfetamina, MDMA).

\section{Potencial de abuso de los antidepresivos}

Desde la década de 1970 se han descrito series de casos que sugieren que algunos antidepresivos pueden tener po- 
tencial de abuso, siendo aquellos con propiedades estimulantes o sedativas los que presentan mayor riesgo. A continuación se describen los antidepresivos con mayor riesgo y con los que se debe tener especial cuidado en los pacientes con TUS (Evans y Sullivan, 2014; Haddad, 1999; Jasinski, Faries, Moore, Schuh, y Allen, 2008; Reeves, Ladner, Perry, Burke, y Laizer, 2015; Volkow et al., 2005)

- IMAO: tranilcipromina y fenelcina han presentado abuso por vía oral relacionado con su estructura similar a las anfetaminas; sobre todo se han descrito series de casos con tranilcipromina.

- Tricíclicos: abuso por vía oral, sobre todo de aquellos que tienen propiedades sedantes y anticolinérgicas. Se han publicado casos con amitriptilina y dotiepina (análogo de la amitriptilina usada en Europa) con el fin de conseguir sensación de euforia.

- Bupropion: abuso por vía intranasal con efectos descritos similares a la cocaína. También se han descrito casos aislados de abuso por vía endovenosa.

- ISRS: existen estudios que indican que se han usado altas dosis por vía oral de fluoxetina para obtener efectos de tipo anfetamínicos en combinación con alcohol o MDMA.

- IRNS: se ha publicado un caso de abuso con venlafaxina que presentó cuadro de abstinencia y requirió ingreso para desintoxicación.

- Tianeptina: es un antidepresivo aprobado en Francia y recientemente en España. Se han publicado casos

Tabla 6. Principales interacciones en el manejo clínico de la depresión dual.

\begin{tabular}{|c|c|c|}
\hline Sustancia/fármaco & Antidepresivo & Efecto \\
\hline \multirow[t]{3}{*}{ Benzodiazepinas } & Tricíclicos & $\uparrow$ concentraciones plasmáticas de desipramina e imipramina \\
\hline & ISRS & Fluoxetina y fluvoxamina \\
\hline & & 个 concentraciones plasmáticas de alprazolam y diazepam \\
\hline \multirow[t]{3}{*}{ Disulfiram } & Tricíclicos & $\begin{array}{l}\uparrow \text { concentraciones plasmáticas de desipramina y amitriptilina por } \downarrow \text { del } \\
\text { metabolismo y aumento de la neurotoxicidad de la combinación }\end{array}$ \\
\hline & IMAO & Tranylcypromina, \\
\hline & & Psicosis confusional con la combinación \\
\hline \multirow[t]{13}{*}{ Opioides } & Tricíclicos & Metadona: \\
\hline & & 个 riesgo de prolongación del intervalo QTC \\
\hline & & $\uparrow$ riesgo de mortalidad si sobredosis \\
\hline & & $\begin{array}{l}\uparrow \text { concentracione splasmáticas de metadona si administración conjunta con } \\
\text { Desipramina: }\end{array}$ \\
\hline & & Doxepina: \\
\hline & & puede inducir delirium durante el SAO \\
\hline & ISRS & Metadona y buprenorfina: \\
\hline & & $\uparrow$ riesgo de síndrome serotoninérgico \\
\hline & & $\uparrow$ concentraciones plasmáticas de metadona por eliminación con Fluvoxamina \\
\hline & IMAO/RIMA & Moclobemida: \\
\hline & & $\uparrow$ efectos de morfina, fentanilo y metadona \\
\hline & Otros & Mirtazapina: \\
\hline & antidepresivos & 个 riesgo de prolongación del intervalo QTc con metadona \\
\hline \multirow[t]{7}{*}{ Alcohol } & Tricíclicos & $\uparrow$ toxicidad del alcohol \\
\hline & & $\downarrow$ función cognitiva \\
\hline & & riesgo of convulsiones (maprotilina) \\
\hline & IMAO & $\uparrow$ efectos del alcohol \\
\hline & & Crisis hipertensivas, por liberación catecolaminas \\
\hline & Otros & \\
\hline & antidepresivos & 个 sedación (trazodona y mirtazapina) \\
\hline \multirow{3}{*}{$\begin{array}{l}\text { Estimulantes } \\
\text { (Cocaína/anfetamina) }\end{array}$} & Tricíclicos & $\downarrow$ craving, y el umbral convulsivo \\
\hline & \& ISRS & $\begin{array}{l}\text { 个 de la frecuencia cardíaca y presión diastólica un } 20-30 \% \text {, } \\
\text { mayor riesgo de arritmia }\end{array}$ \\
\hline & IMAOs & Contraindicación absoluta \\
\hline
\end{tabular}

Nota. SAO: Síndrome de Abstinencia a Opiáceos; IMAOs: Inhibidores de la monoaminooxidasa; ISRS :inhibidores selectivos de la recaptación de serotonina; RIMA: inhibidor reversible de la MAO-A. 
de abuso por vía oral para obtener un efecto psicoestimulante.

- Amineptina: el abuso por vía oral presenta efectos de tipo estimulante.

\section{Tratamientos psicológicos}

El tratamiento de la depresión dual con terapia cognitivo- conductual (TCC) está bien reconocido. Sin embargo, en la práctica clínica todavía no se aplica habitualmente a pesar de los datos disponibles sobre su eficacia.

Actualmente se dispone de diversos tratamientos combinados para DM y TUS incluyendo tratamientos psicoterapéuticos como adyuvantes o alternativos al tratamiento farmacológico. Recientemente una revisión sistemática con metaanálisis ha evaluado la eficacia de la TCC y la Intervención Motivacional sobre la DM en pacientes con TUS por alcohol vs tratamiento habitual (Riper et al., 2014). Los autores observaron que en ambos casos las intervenciones mostraron un leve efecto clínicamente significativo tanto para la disminución de los síntomas de depresión como para la disminución del consumo de alcohol, aunque el tamaño del efecto fue inferior comparado con el obtenido con los tratamientos farmacológicos. Por otra parte, en el proyecto Building Recovery by Improving Goals, Habits, and Thoughts (BRIGHT), en el que se comparó el tratamiento residencial del TUS con el tratamiento residencial del TUS junto con TCC, se observaron mejores resultados clínicos con mayor adherencia al tratamiento y mayor mejoría de los síntomas depresivos en los pacientes que recibieron además TCC (Watkins et al., 2011).

\section{Protocolo de Intervención}

\section{Evaluación diagnóstica}

Dado que los fármacos antidepresivos han demostrado mayor eficacia en los trastornos independientes que en los inducidos, uno de los puntos clave para el tratamiento es una buena aproximación diagnóstica, como ya se ha comentado previamente. La literatura médica indica que las entrevistas estructuradas son la mejor herramienta para establecer estos diagnósticos y que la entrevista PRISM (Psychiatric Research Interview for Substance and Mental Disorders) es la más apropiada para ello. Además de esto, también es importante valorar la intensidad del episodio para plantear el inicio de tratamiento con antidepresivos.

\section{Ámbito de tratamiento}

En el ámbito ambulatorio a veces no es posible mantener a los pacientes abstinentes ni orientar una reducción significativa en el consumo. Debe considerarse el ingreso hospitalario, ya sea urgente o programado, incluso en pacientes con sintomatología depresiva moderada, independientemente de si es inducida o primaria, para estabilizar al paciente.

\section{Tratamiento del TUS}

Aun existiendo sintomatología depresiva, no se puede olvidar el tratamiento del TUS y se deben iniciar intervenciones psicosociales y farmacológicas para disminuir el consumo de sustancias (por ejemplo, naltrexona o nalmefene para la dependencia de alcohol, metadona o buprenorfina-naloxona, para el tratamiento de la dependencia de opiáceos). Para reducir el riesgo de recaídas a largo plazo de los dependientes de alcohol y otras drogas, es importante la valoración y el tratamiento de la depresión mayor.

\section{Tratamiento farmacológico de la depresión}

Debe tenerse en consideración el tratamiento con antidepresivos no ISRS en los pacientes. Añadir un perfil más dopaminérgico y noradrenérgico o mecanismos mixtos de
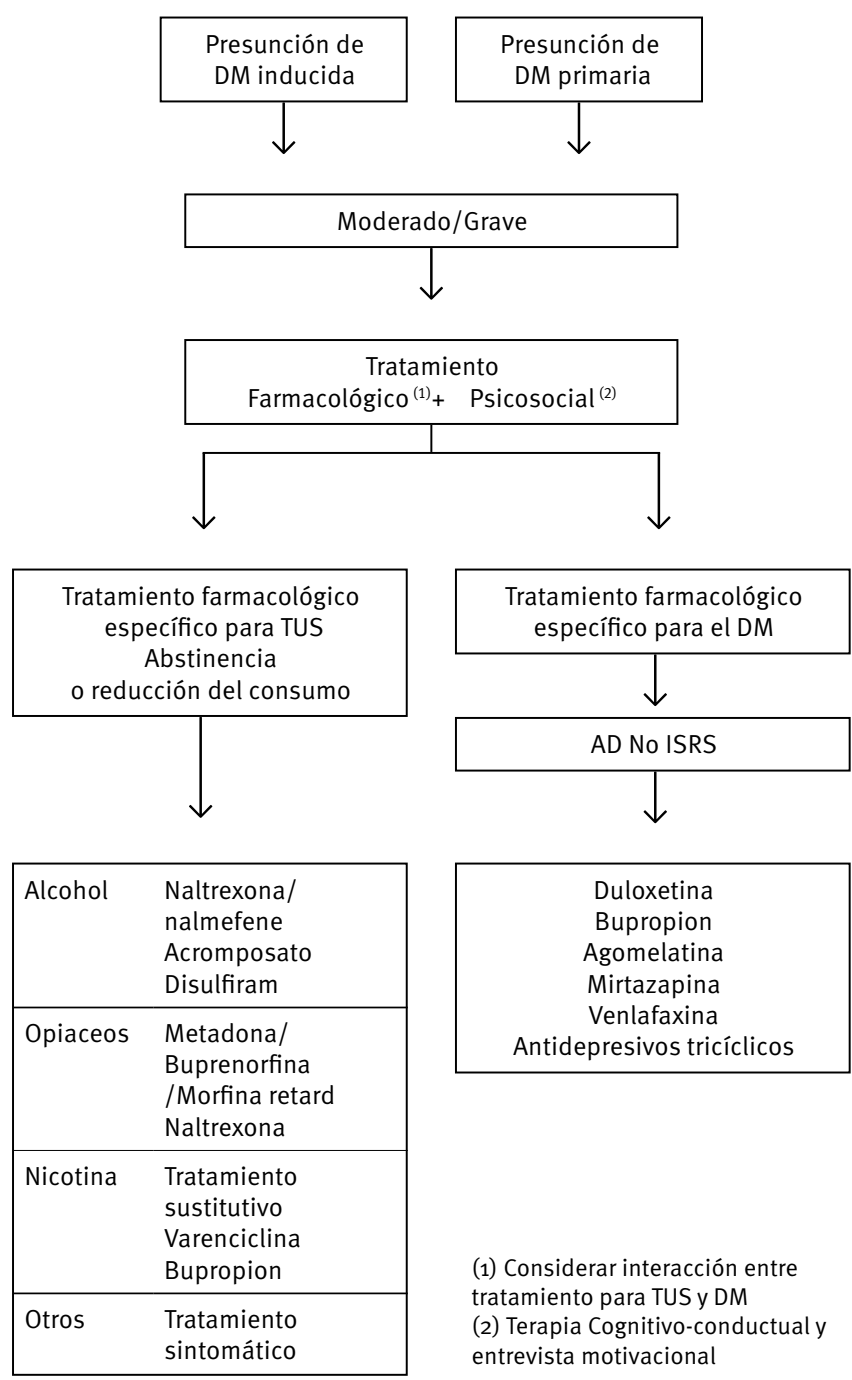

Figura 1. Algoritmo terapéutico para el tratamiento de la depresión mayor y el trastorno por uso de sustancias. 
acción parece ser más eficaz. En la Figura 1 se muestra un algoritmo terapéutico para el tratamiento de la patología dual DM-TUS.

Finalmente, es necesario resaltar que, pese la elevada prevalencia de DM en pacientes con TUS, las evidencias disponibles en cuanto al mejor tratamiento son escasas. La investigación futura debería proponer ensayos controlados para analizar el perfil de eficacia, seguridad e interacciones de los nuevos antidepresivos disponibles.

\section{Tratamiento paralelo, secuencial o integrado}

Es importante destacar que en la mayoría de los países existen dos redes separadas para el tratamiento de las enfermedades mentales y para el tratamiento de los TUS. Esto implica que los pacientes con patología dual muy frecuentemente son tratados en dos dispositivos: el de salud mental y el de adicciones (modelo de tratamiento paralelo). Además, en muchos casos la abstinencia a sustancias es un requisito fundamental previo al tratamiento de la depresión (modelo de tratamiento secuencial). Actualmente, estos modelos de tratamiento se recomienda que sean sustituidos por el denominado modelo integrado que implica una aproximación simultánea y coordinada de ambos trastornos, el adictivo y el afectivo, con el fin de mejorar la adherencia y la efectividad del tratamiento (Torrens, Rossi, Martinez-Riera, Martinez-Sanvisens, y Bulbena, 2012)

\section{Conclusiones}

La comorbilidad de DM y TUS es frecuente y todos aquellos pacientes afectados por un trastorno dual presentarán mayor gravedad psicopatológica y médica, así como peor funcionamiento social. Es de elevada relevancia tratar la DM y el TUS de forma simultánea basándonos en el modelo integrado y no aproximarnos al tratamiento de ambas patologías de forma separada o secuencial. También es de extrema prioridad seguir investigando en la neurobiología de los mecanismos de acción implicados en los trastornos duales con el fin de desarrollar tratamientos y estrategias de prevención más eficaces.

\section{Reconocimientos}

Esta revisión se ha realizado en parte gracias a ayudas para investigación de la European Commission- Directorate General for Research and Innovation (Horizon 2020) (Creating medically driven integrative bioinformatics applications focused on oncology, CNS disorders and their comorbidities: MedBioinformatics $\mathrm{N}^{\mathrm{o}}$ contrato: 634143) y del Instituto de Salud Carlos III (Red de Trastornos Adictivos, UE-FEDER 2012, RD16/0017/0010). También agradecemos a Warren Meredith por leer el manuscrito y sus sugerencias aportadas.

\section{Conflictos de interés}

Todos los autores declaran que no tienen ningún conflicto de interés.

\section{Referencias}

Agosti, V. y Levin, F. R. (2006). One-year follow-up study of suicide attempters treated for drug dependence. The American Journal on Addictions, 15, 293-296. http://doi. org/10.1080/10550490600754333.

American Psychiatric Association, APA (1994). DSM-IV. Manual diagnóstico y estadístico de los trastornos mentales. Barcelona: Masson.

American Psychiatric Association, APA (2002). DSM-IV-TR. Manual diagnóstico y estadístico de los trastornos mentales. Texto Revisado. Barcelona: Masson.

American Psychiatric Association, APA. (2013). DSM-V. Ma nual diagnóstico y estadístico de los trastornos mentales. Barcelona: Masson.

Arias, F., Szerman, N., Vega, P., Mesias, B., Basurte, I., Morant, C., ... Babín, F. (2013). Madrid study on the prevalence and characteristics of outpatients with dual pathology in community mental health and substance misuse services. Adicciones, 25, 118-27. doi:10.1080/17523281.2 012.748674 .

Belmaker, R. H. y Agam, G. (2008). Major depressive disorder. The New England Journal of Medicine, 358, 55-68. doi:10.1056/NEJMra073096.

Boden, J. M. y Fergusson, D. M. (2011). Alcohol and depression. Addiction, 106, 906-914. Doi:10.1111/j.13600443.2010.03351.x.

Brady, K. T. y Sinha, R. (2005). Co-occurring mental and substance use disorders: The neurobiological effects of chronic stress. American Journal of Psychiatry, 162, 14831493. doi:10.1176/appi.ajp.162.8.1483.

Chou, R., Cruciani, R. A., Fiellin, D. A., Compton, P., Farrar, J. T., Haigney, M. C., ... Heart Rhythm Society. (2014). Methadone safety: a clinical practice guideline from the American Pain Society and College on Problems of Drug Dependence, in collaboration with the Heart Rhythm Society. The Journal of Pain: Official Journal of the American Pain Society, 15, 321-337. doi:10.1016/j. jpain.2014.01.494.

Davis, L., Uezato, A., Newell, J. M. y Frazier, E. (2008). Major depression and comorbid substance use disorders. Current Opinion in Psychiatry, 21, 14-18. doi:10.1097/ YCO.0b013e3282f32408.

EMCDDA. (2015). Comorbidity of substance use and mental disorders in Europe. Luxembourg. doi:10.2810/532790.

Evans, E. A. y Sullivan, M. A. (2014). Abuse and misuse of antidepressants. Substance Abuse and Rehabilitation, 5, 107-120. doi:10.2147/SAR.S37917.

Gutiérrez-Sacristán, A., Grosdidier, S., Valverde, O., Torrens, M., Bravo, À., Piñero, J., ... Furlong, L. I. (2015). 
PsyGeNET: a knowledge platform on psychiatric disorders and their genes. Bioinformatics (Oxford, England), 31, 3075-3077. doi:10.1093/bioinformatics/btv301.

Haddad, P. (1999). Do antidepressants have any potential to cause addiction? Journal of Psychopharmacology (Oxford, England), 13, 300-307.

Hasin, D. S., Trautman, K. D., Miele, G. M., Samet, S., Smith, M. y Endicott, J. (1996). Psychiatric Research Interview for Substance and Mental Disorders (PRISM): reliability for substance abusers. The American Journal of Psychiatry, 153, 1195-201. doi.org/10.1176/ajp.153.9.1195.

Jasinski, D. R., Faries, D. E., Moore, R. J., Schuh, L. M. y Allen, A. J. (2008). Abuse liability assessment of atomoxetine in a drug-abusing population. Drug and Alcohol Dependence, 95, 140-146. doi:10.1016/j.drugalcdep.2008.01.008.

Kao, D., Bucher Bartelson, B., Khatri, V., Dart, R., Mehler, P. S., Katz, D. y Krantz, M. J. (2013). Trends in reporting methadone-associated cardiac arrhythmia, 19972011: an analysis of registry data. Annals of Internal Medicine, 158, 735-740. doi:10.7326/0003-4819-158-10201305210-00008.

Krishnan, V. y Nestler, E. J. (2010). Linking molecules to mood: new insight into the biology of depression. The American Journal of Psychiatry, 167, 1305-1320. doi:10.1176/appi.ajp.2009.10030434.

Lai, H. M. X., Cleary, M., Sitharthan, T. y Hunt, G. E. (2015). Prevalence of comorbid substance use, anxiety and mood disorders in epidemiological surveys, 19902014: A systematic review and meta-analysis. Drug and Alcohol Dependence, 154, 1-13. doi:10.1016/j.drugalcdep.2015.05.031.

Landheim, A. S., Bakken, K. y Vaglum, P. (2006). Impact of comorbid psychiatric disorders on the outcome of substance abusers: a six year prospective follow-up in two Norwegian counties. BMC Psychiatry, 6, 44. doi:10.1186/1471-244X-6-44.

Langås, A.-M., Malt, U. F. y Opjordsmoen, S. (2013). Independent versus substance-induced major depressive disorders in first-admission patients with substance use disorders: an exploratory study. Journal of Affective Disorders, 144, 279-283. doi:10.1016/j.jad.2012.10.008.

Levin, F. R., Mariani, J., Brooks, D. J., Pavlicova, M., Nunes, E. V, Agosti, V., ... Carpenter, K. M. (2013). A randomized double-blind, placebo-controlled trial of venlafaxine-extended release for co-occurring cannabis dependence and depressive disorders. Addiction (Abingdon, England), 108, 1084-1094. doi:10.1111/add.12108.

Marmorstein, N. R. (2011). Associations between subtypes of major depressive episodes and substance use disorders. Psychiatry Research, 186, 248-253. doi:10.1016/j. psychres.2010.10.003.

Martin-Santos, R., Fonseca, F., Domingo-Salvany, A., Gines, J. M., Imaz, M. L., Navines, R., ... Torrens, M. (2006).
Dual Diagnosis in the Psychiatric Emergency Room in Spain. The European Journal of Psychiatry, 20, 147-156.

Mueller, T. I., Lavori, P. W., Keller, M. B., Swartz, A., Warshaw, M., Hasin, D., ... Akiskal, H. (1994). Prognostic effect of the variable course of alcoholism on the 10-year course of depression. The American Journal of Psychiatry, 151, 701-706. doi:10.1176/ajp.151.5.701.

Nestler, E. J. y Carlezon, W. A. (2006). The Mesolimbic Dopamine Reward Circuit in Depression. Biological Psychiatry, 59, 1151-1159. doi:10.1016/j.biopsych.2005.09.018.

Nunes, E. V. y Levin, F. R. (2004). Treatment of depression in patients with alcohol or other drug dependence: a meta-analysis. JAMA : The Journal of the American Medical Association, 291, 1887-1896. doi:org/10.1001/ jama.291.15.1887.

Pani, P. P., Vacca, R., Trogu, E., Amato, L. y Davoli, M. (2010). Pharmacological treatment for depression during opioid agonist treatment for opioid dependence. The Cochrane Database of Systematic Reviews, 9, CD008373. doi:10.1002/14651858.CD008373.pub2.

Pettinati, H. M., O'Brien, C. P. y Dundon, W. D. (2013). Current status of co-occurring mood and substance use disorders: a new therapeutic target. The American Journal of Psychiatry, 170, 23-30. doi:10.1176/appi. ajp.2012.12010112.

Reeves, R. R., Ladner, M. E., Perry, C. L., Burke, R. S. y Laizer, J. T. (2015). Abuse of medications that theoretically are without abuse potential. Southern Medical Journal, 108, 151-157. doi:10.14423/SMJ.0000000000000256.

Riper, H., Andersson, G., Hunter, S. B., de Wit, J., Berking, M. y Cuijpers, P. (2014). Treatment of comorbid alcohol use disorders and depression with cognitive-behavioural therapy and motivational interviewing: A meta-analysis. Addiction, 109, 394-406. doi:10.1111/add.12441.

Samet, S., Fenton, M. C., Nunes, E., Greenstein, E., Aharonovich, E. y Hasin, D. (2013). Effects of independent and substance-induced major depressive disorder on remission and relapse of alcohol, cocaine and heroin dependence. Addiction, 108, 115-123. doi:10.1111/j.13600443.2012.04010.x.

San, L., Arranz, B. y Grupo de Expertos de la Guía de Práctica Clínica de Patología Dual. (2016). Guía de práctica clínica para el tratamiento de patología dual en población adulta. Adicciones, 28, 3-5. doi:10.20882/adicciones.784.

Schuckit, M. A. (2006). Comorbidity between substance use disorders and psychiatric conditions. Addiction, 101, 76-88. doi:10.1111/j.1360-0443.2006.01592.x.

Schuckit, M. A., Smith, T. L. y Kalmijn, J. (2013). Relationships among independent major depressions, alcohol use, and other substance use and related problems over 30 years in 397 families. Journal of Studies on Alcohol and Drugs, 74, 271-279.

Szerman, N., Arias, F., Vega, P., Babín, F. D. A., Mesías, B., Basurte, I., ... Grupo de Investigación del estudio piloto 
sobre la prevalencia de patología dual en pacientes en tratamiento en la Comunidad de Madrid. Estudio piloto sobre la prevalencia de patología dual en pacientes en tratamiento en la Comunidad de Madrid, Adicciones, 23, 249-255.

Torrens, M., Fonseca, F., Mateu, G. y Farre, M. (2005). Efficacy of antidepressants in substance use disorders with and without comorbid depression: A systematic review and meta-analysis. Drug and Alcohol Dependence, 78, 1-22. doi:10.1016/j.drugalcdep.2004.09.004.

Torrens, M., Gilchrist, G. y Domingo-Salvany, A. (2011). Psychiatric comorbidity in illicit drug users: Substance-induced versus independent disorders. Drug and Alcohol Dependence, 113, 147-156. doi:10.1016/j.drugalcdep.2010.07.013.

Torrens, M., Martínez-Sanvisens, D., Martínez-Riera, R., Bulbena, A., Szerman, N. y Ruiz, P. (2011). Dual Diagnosis: Focusing on Depression and Recommendations for Treatment. Addictive Disorders y Their Treatment, 10, 50-59. doi:10.1097/ADT.0b013e318215f322.

Torrens, M. y Rossi, P. (2015). Mood disorders and addiction. In G. Dom y F. Moggi (Eds.), Co-Occurring Addictive and Psychiatric Disorders: A Practice-Based Handbook from A European Perspective (1st ed., pp. 103-117). Berlin, Heidelberg: Springer Berlin Heidelberg. doi:10.1007/9783-642-45375-5_8.

Torrens, M., Rossi, P. C., Martinez-Riera, R., Martinez-Sanvisens, D. y Bulbena, A. (2012). Psychiatric co-morbidity and substance use disorders: Treatment in parallel systems or in one integrated system. Substance Use and Misuse, 47, 1005-1014. doi:10.3109/10826084.2012.663296.

Valverde, O., Célérier, E., Baranyi, M., Vanderhaeghen, P., Maldonado, R., Sperlagh, B., ... Ledent, C. (2009). GPR3 receptor, a novel actor in the emotional-like responses. PloS One, 4, e4704. doi:10.1371/journal.pone.0004704.

Valverde, O. y Torrens, M. (2012). CB1 receptor-deficient mice as a model for depression. Neuroscience, 204, 193206. doi:10.1016/j.neuroscience.2011.09.031.

Volkow, N. D., Wang, G.-J., Fowler, J. S., Learned-Coughlin, S., Yang, J., Logan, J., ... Xu, Y. (2005). The slow and long-lasting blockade of dopamine transporters in human brain induced by the new antidepressant drug radafaxine predict poor reinforcing effects. Biological Psychiatry, 57, 640-646. doi:10.1016/j.biopsych.2004.12.007.

Watkins, K. E., Hunter, S. B., Hepner, K. A., Paddock, S. M., de la Cruz, E., Zhou, A. J. y Gilmore, J. (2011). An effectiveness trial of group cognitive behavioral therapy for patients with persistent depressive symptoms in substance abuse treatment. Archives of General Psychiatry, 68, 577-584. doi:10.1001/archgenpsychiatry.2011.53.

Wise, R. A. (1989). Opiate reward: Sites and substrates. Neuroscience and Biobehavioral Reviews, 13, 129-133. doi:10.1016/S0149-7634(89)80021-1. 